\title{
Antiviral mechanism and biochemical basis of the human APOBEC3 family
}

\author{
Mayumi Imahashi ${ }^{1,2}$, Masaaki Nakashima ${ }^{1,3}$ and Yasumasa Iwatani ${ }^{1,2}$ * \\ 1 Clinical Research Center, National Hospital Organization Nagoya Medical Center, Nagoya, Japan \\ ${ }^{2}$ Graduate School of Medicine, Nagoya University, Nagoya, Japan \\ ${ }^{3}$ Graduate School of Engineering, Nagoya University, Nagoya, Japan
}

Edited by:

Atsushi Koito, Kumamoto University, Japan

\section{Reviewed by:}

Harold Charles Smith, University of

Rochester, USA

Hiroshi Matsuo, University of

Minnesota, USA

${ }^{*}$ Correspondence:

Yasumasa Iwatani, Laboratory of Infectious Diseases, Department of Microbiology and Immunology, Clinical Research Center, National Hospital Organization Nagoya Medical Center, 4-1-1 San-no-Maru, Naka-ku,

Nagoya, Aichi 460-0001, Japan.

e-mail: iwataniy@nnh.hosp.go.jp

\begin{abstract}
The human APOBEC3 (A3) family ( $\mathrm{A}, \mathrm{B}, \mathrm{C}, \mathrm{DE}, \mathrm{F}, \mathrm{G}$, and $\mathrm{H}$ ) comprises host defense factors that potently inhibit the replication of diverse retroviruses, retrotransposons, and the other viral pathogens. HIV-1 has a counterstrategy that includes expressing the Vif protein to abrogate $\mathrm{A} 3$ antiviral function. Without Vif, A3 proteins, particularly APOBEC3G (A3G) and APOBEC3F (A3F), inhibit HIV-1 replication by blocking reverse transcription and/or integration and hypermutating nascent viral cDNA. The molecular mechanisms of this antiviral activity have been primarily attributed to two biochemical characteristics common to $A 3$ proteins: catalyzing cytidine deamination in single-stranded DNA (ssDNA) and a nucleic acid-binding capability that is specific to ssDNA or ssRNA. Recent advances suggest that unique property of $\mathrm{A} 3 \mathrm{G}$ dimer/oligomer formations, is also important for the modification of antiviral activity. In this review article we summarize how A3 proteins, particularly A3G, inhibit viral replication based on the biochemical and structural characteristics of the A3G protein.
\end{abstract}

Keywords: antiviral, APOBEC3, APOBEC3G, cytidine deaminase, HIV, retrovirus, reverse transcription, Vif

\section{INTRODUCTION}

Productive infections of primary human lymphocytes, monocytes, and certain T-cell lines by HIV-1 require a virally encoded gene product, Vif (originally named "Sor" or "A"; Fisher et al., 1987; Strebel et al., 1987). In early work on Vif, vif-deficient virions produced in non-permissive cells were found to be significantly impaired in their ability to complete reverse transcription (Sova and Volsky, 1993; von Schwedler et al., 1993), and they were 100- to 1000-fold less infectious than wild type (WT) virions (Fisher et al., 1987; Strebel et al., 1987; Fouchier et al., 1996). Sheehy et al. (2002) identified $A 3 G$ as the cellular enzyme that restricts the replication of vif-deficient HIV-1.

The human A3G protein is a cellular cytidine deaminase that belongs to the APOBEC3 family, which comprises seven members (A3A, B, C, DE, F, G, and H; LaRue et al., 2009). These proteins contain one $(\mathrm{A} 3 \mathrm{~A}, \mathrm{~A} 3 \mathrm{C}$, and $\mathrm{A} 3 \mathrm{H})$ or two (A3B, $\mathrm{A} 3 \mathrm{DE}, \mathrm{A} 3 \mathrm{~F}$, and $\mathrm{A} 3 \mathrm{G}$ ) zinc-cluster domains with the consensus sequence (H/C)XE(X) ${ }_{23-28}$ CXXC (Wedekind et al., 2003). Among the APOBEC 3 family members, $\mathrm{A} 3 \mathrm{G}$ is the most potent inhibitor of HIV- 1 but only in the absence of Vif. HIV-1 Vif counteracts A3G by promoting its polyubiquitination through the recruitment of a Cullin5-based E3 ubiquitin ligase complex (Yu et al., 2003), which targets A3G proteins for rapid proteasomal degradation in infected cells.

The specific $A 3 \mathrm{G}$ degradation is determined by the capability of Vif to bind with A3G in the E3 ubiquitin ligase complex (reviewed in Kitamura et al., 2011). The region in A3G responsible for HIV-1 Vif interaction was identified by the studies on the species specificity of Vif-mediated A3G degradation, which is determined by a single amino acid difference in human A3G, D128 versus K128 in the A3G of African green monkeys (Bogerd et al., 2004; Mangeat et al., 2004; Schrofelbauer et al., 2004; Xu et al., 2004). Subsequent mutational analyses have confirmed that the 128DPD130 motif of A3G, located near the zinc-coordinating residues of NTD, is crucial for direct binding to HIV-1 Vif (Huthoff and Malim, 2007; Russell et al., 2009; Lavens et al., 2010). This motif is just downstream of residues 124YYFW127, which are involved in A3G's ability to bind nucleic acids (Huthoff and Malim, 2007).

The primary mechanism by which A3G inhibits vif-deficient HIV-1 replication requires its expression in virus producer cells and its incorporation into virions (Mariani et al., 2003; Marin et al., 2003; Sheehy et al., 2003; Stopak et al., 2003; Svarovskaia et al., 2004). During reverse transcription in the target cells, the virion-packaged A3G deaminates cytidine to uridine in the viral minus-strand DNA (Harris et al., 2003a; Lecossier et al., 2003; Mangeat et al., 2003; Zhang et al., 2003; Suspène et al., 2004; Yu et al., 2004). Subsequent incorporation of adenines instead of guanines in the plus-strand results in extensive G-to-A hypermutation and inactivation of the viral genome. Shortly after A3G was suggested as the key restriction factor against vif-deficient HIV-1, it was proposed that A3G-mediated deamination might be a lethal trigger, eventually leading to the degradation of reversetranscribed viral DNA through a base-excision pathway and/or the reduced replication of progeny viruses by introducing premature stop codons and/or amino acid changes (Cullen, 2003; Goff, 2003; Harris et al., 2003a,b; KewalRamani and Coffin, 2003). Indeed, the catalytic center of the A3G protein is clearly essential for its antiviral functions (Mangeat et al., 2003; Navarro et al., 2005; Iwatani et al., 2006; Browne et al., 2009). However, several lines of recent evidence have indicated that the catalytic activity of A3G is not 
sufficient to explain its full antiviral activity. What is the fundamental mechanism(s) of A3G antiviral activity that explains the observation by von Schwedler et al. (1993), that the reverse transcription of vif-deficient HIV-1 is impaired when produced from A3G-expressing "non-permissive" cells?

\section{BIOCHEMICAL PROPERTIES OF A3G}

The zinc coordination of A3 family proteins is mediated by a histidine and two cysteines, which form a catalytic center for cytidine deaminase activity. In A3G, the zinc-binding motif at the C-terminal domain (CTD) is primarily associated with cytidine deaminase catalysis whereas the N-terminal domain (NTD) does not catalyze deamination (Figure 1A; Haché et al., 2005; Navarro et al., 2005; Iwatani et al., 2006; Friew et al., 2009). The A3G enzyme converts deoxycytidine ( $\mathrm{dC}$ ) residues to deoxyuridine $(\mathrm{dU})$, and acts preferentially on residues that are preceded by another $\mathrm{dC}$, with a much higher preference for the $5^{\prime}$-CCCA$3^{\prime}$ sequence in single-stranded DNA (ssDNA; Beale et al., 2004; Suspène et al., 2004; Yu et al., 2004). During retroviral reverse transcription, $\mathrm{A} 3 \mathrm{G}$ deaminates $\mathrm{dC}$ to $\mathrm{dU}$ in the viral minus-stranded DNA, and the subsequent incorporation of deoxyadenines (dA) instead of deoxyguanines $(\mathrm{dG})$ in the plus-strand results in G-toA hypermutation of the nascent viral DNA (Harris et al., 2003a; Lecossier et al., 2003; Mangeat et al., 2003; Zhang et al., 2003; Suspène et al., 2004; Yu et al., 2004). The ssDNA-specific deamination by $\mathrm{A} 3 \mathrm{G}$ appears to be determined by a structural groove, presumably accommodating ssDNA, that positions the cytosine for deamination (Chen et al., 2008; Holden et al., 2008).

The nucleic acid-binding property of $\mathrm{A} 3 \mathrm{G}$ is also a major biochemical feature. The minimum unit of $\mathrm{A} 3 \mathrm{G}$ for binding to ssDNA is a monomer (Chelico et al., 2010) and/or a dimer (Bennett et al.,
2008), as illustrated in Figure 1B. The apparent equilibrium dissociation constant (Kd) for A3G (to ssDNA) is between 52 and $238 \mathrm{nM}$ (Chelico et al., 2006; Iwatani et al., 2006, 2007), whereas the Kd for HIV-1 nucleocapsid protein (NC) binding to RNA is approximately 23-320 nM (Shubsda et al., 2002; Levin et al., 2005), suggesting that the nucleic acid-binding affinity of A3G is as high as that of NC. A3G binds preferentially to ssDNA or ssRNA (Figure 1B; Yu et al., 2004; Iwatani et al., 2006; Shlyakhtenko et al., 2011), especially dT or dU residues of ssDNA and AU-rich regions in ssRNA, respectively (Jarmuz et al., 2002; Iwatani et al., 2006). Interestingly, the substrate specificities and nucleotide preferences of the $\mathrm{A} 3 \mathrm{G}$ protein differ for its deaminase and nucleic acid-binding activities, as is the case for APO1 (Anant et al., 1995; Navaratnam et al., 1995; Anant and Davidson, 2000). Because mutations that disrupt zinc coordination at the NTD, such as the substitution of the $\mathrm{C} 100$ residue with a serine, abrogate the nucleic acid-binding affinity of A3G (Navarro et al., 2005; Iwatani et al., 2006), some local conformation near the zinc coordination of NTD might be responsible for its recognition of single-stranded nucleotides.

The formation of an A3G homo-multimer is the third unique feature of A3G. The intrinsic propensity of A3G multimerization has been verified by biochemical and structural studies (Jarmuz et al., 2002; Navarro et al., 2005; Burnett and Spearman, 2007; Bennett et al., 2008; Bulliard et al., 2009; Friew et al., 2009; Huthoff et al., 2009; Chelico et al., 2010; McDougall et al., 2011). Because the full-length $\mathrm{A} 3 \mathrm{G}$ structure has not been determined, the A3G interface for multimerization remain unclear. However, structural analyses by SAXS (small-angle X-ray scattering), coimmunoprecipitation assays (Wedekind et al., 2006; Bennett et al., 2008), and X-ray crystallography (Shandilya et al., 2010) have
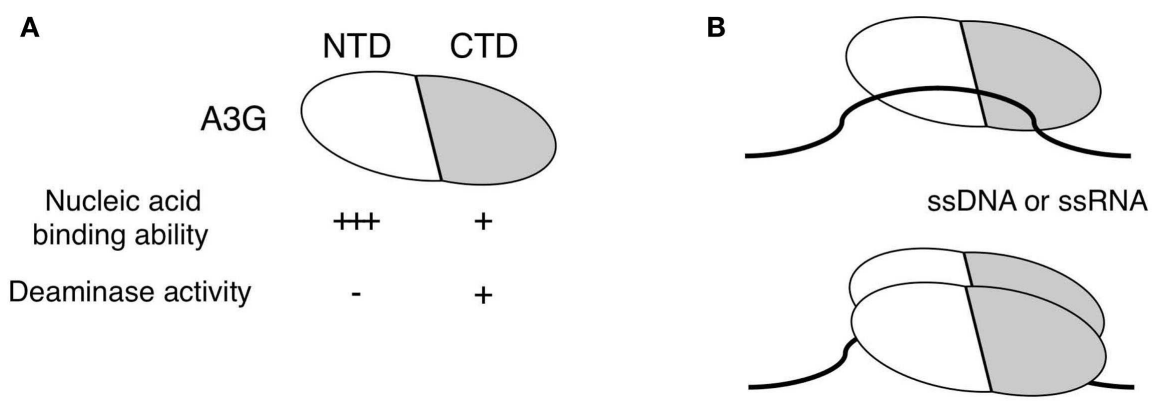

C

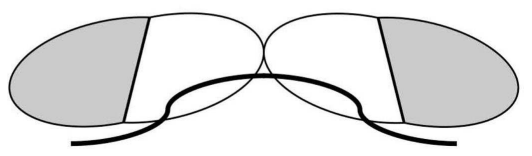

Head-Head

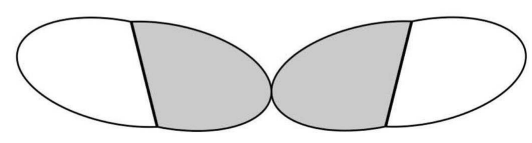

Tail-Tail
FIGURE 1 | Biochemical characteristics of A3G. (A) A3G consists of an NTD and CTD. The NTD is responsible for the nucleic acid-binding affinity of $A 3 G$ and has no detectable deaminase activity. In contrast, the CTD is solely involved in deaminase activity and has less affinity for nucleic acids than the NTD. (B) A3G specifically binds to single-stranded
DNA (ssDNA) or RNA (ssRNA) but not to double-stranded nucleotides. A monomeric and/or dimeric A3G bind to ssDNA or ssRNA as the minimum unit. (C) The $A 3 G$ protein forms homodimers or higher-order homo-oligomers through interactions between its NTDs (head-head) or CTDs (tail-tail). 
demonstrated an interaction between the A3G CTDs (tail-tail), as illustrated in Figure 1C. In addition, homo-dimerization through the NTDs also occurs (head-head), as shown in Figure 1C, and this interaction appears to depend on the presence of RNA (Friew et al., 2009; Huthoff et al., 2009). These observations were supported by an analytical ultracentrifugation study that showed a predominant dimer form of A3G in equilibrium with minor monomeric and tetrameric species under RNA-depleted conditions (Salter et al., 2009).

Chelico et al. (2006, 2008, 2010) have used an in vitro system to demonstrate that $\mathrm{A} 3 \mathrm{G}$ has a $3^{\prime}$ to $5^{\prime}$ catalytic orientation specificity for the deamination of naked ssDNA (Feng and Chelico, 2011). The preferred asymmetric direction for A3G catalysis likely yields an approximately 30-nt "dead" zone located at the $3^{\prime}$ end of ssDNA that is much less efficiently deaminated by A3G (Chelico et al., 2008, 2010). However, we need further investigations on how significant the length of dead zone is because the central CCC motif of $~ 40-n t$ ssDNA can be deaminated efficiently by $\mathrm{A} 3 \mathrm{G}$ in our or the other in vitro deamination assay systems (Beale et al., 2004; Yu et al., 2004; Iwatani et al., 2006). The formation of tetramers and higher-order homo-oligomers of A3G on ssDNA is required for efficient deamination (McDougall et al., 2011).

\section{ANTIVIRAL MECHANISMS OF A3G AGAINST VIF-DEFICIENT HIV-1}

A3G exerts its inhibitory activity by being encapsidated into virus particles of vif-deficient HIV-1. During the subsequent infection cycle, A3G has been proposed to interfere with reverse transcription and/or integration through one or more molecular mechanisms (Figure 2). Based on whether the enzymatic activity is involved or not, there are two separable mechanisms, i.e., deaminase-dependent and -independent mechanisms.

Although the catalytic center of A3G is clearly critical for its antiviral effect (Mangeat et al., 2003; Navarro et al., 2005; Iwatani et al., 2006; Browne et al., 2009), the precise molecular mechanisms underlying the inhibition of the further processing of A3G-deaminated DNA products in cells remain unclear. A3Gmediated hypermutation of viral genomes is clearly detrimental to further spreading the infection because mutations in the viral structural and/or the regulatory genes may trigger defects in the production of infectious progeny virus ("1" in Figure 2). For example, because the preferred sequences of A3G include TGG (a codon for tryptophan within the viral orf), many G-to-A mutations may incidentally produce premature stop codons, such as TAG (or TGA), resulting in viral inactivation (Simon et al., 2005; Pace et al., 2006).

In its second mechanism, A3G reduces the efficiency and specificity of primer tRNA processing and removal, resulting in proviral DNA ends that are aberrant substrates for integration and plusstrand DNA transfer (Luo et al., 2007; Mbisa et al., 2007; “2" in Figure 2). In this mechanism, the presumed deamination sites are located at the plus primer-binding site (PBS), which is annealed by the tRNA. Considering the biochemical characteristics of A3G, it remains unclear how the $\mathrm{A} 3 \mathrm{G}$ enzyme deaminates cytidine residues on the DNA/RNA duplex and near the $3^{\prime}$ end of the plusstrand transfer donor DNA, which is supposed to be a presumed "dead" zone for A3G-mediated deamination (Chelico et al., 2008, 2010).

It was hypothesized that the antiviral functions of A3G might be associated with the uracilation of the nascent reverse transcripts (Harris et al., 2003a; Zhang et al., 2003), resulting in their degradation through the activity of cellular DNA glycosylases, e.g., UDG2 (uracil DNA glycosidase 2) and SMUG1 (single-strand selective monofunctional uracil DNA glycosylase). However, several groups have revealed that neither uracil DNA glycosidase affected the antiviral effect of A3G (Kaiser and Emerman, 2006; Mbisa et al., 2007; Langlois and Neuberger, 2008), although one study showed that UDG2 is involved in the degradation of nascent reverse transcripts (Yang et al., 2007). In addition, we cannot exclude the possibility that other unidentified DNA repair enzymes might participate in the degradation mechanism. Therefore, further studies will be required to elucidate the potential factors that precede the degradation of uracilated DNA following A3G-mediated deamination ("4" in Figure 2).

Earlier studies on A3G suggested that G-to-A hypermutation resulting in lethal mutations was the sole basis of the $\mathrm{A} 3 \mathrm{G}$ antiviral mechanism. However, more recent studies have demonstrated that the catalytic activity of A3G may not wholly determine its molecular mechanism, i.e., a deaminase-independent mechanism might also be involved in A3G antiviral activity: (i) mutations of the catalytic center do not completely abolish antiviral activity against HIV-1 (Navarro et al., 2005; Iwatani et al., 2006; Holmes et al., 2007; Luo et al., 2007; Miyagi et al., 2008); (ii) A3G inhibits replication of hepatitis B virus without detecting significant G-toA hypermutation (Turelli et al., 2004; Bonvin and Greeve, 2007; Nguyen et al., 2007; reviewed in Bonvin and Greeve, 2008); and (iii) other A3 proteins block the replication of HIV-1 (Luo et al., 2007; Miyagi et al., 2010), mouse mammary tumor virus (Okeoma et al., 2007), murine leukemia virus (Takeda et al., 2008), and parvovirus adeno-associated virus (Narvaiza et al., 2009) and retrotransposition of LINE-1 and Alu (Bogerd et al., 2006; Muckenfuss et al., 2006; Stenglein and Harris, 2006; Wissing et al., 2011) despite the absence of editing activity.

Several groups have reported that the deaminase-independent mechanisms of reverse transcription inhibition would involve interference with tRNA primer annealing, initiation and elongation of DNA synthesis, and minus-/plus-strand transfer reactions (Guo et al., 2006, 2007; Iwatani et al., 2007; Li et al., 2007; Luo et al., 2007; Mbisa et al., 2007; Anderson and Hope, 2008; Bishop et al., 2008; Zhang et al., 2008). Using enzymatically active recombinant $\mathrm{A} 3 \mathrm{G}$ and the in vitro reconstituted systems of HIV-1 reverse transcription, it has been demonstrated that A3G blocks all RTcatalyzed DNA elongation processes in a deaminase-independent manner, although the protein does not significantly interfere with tRNA primer placement (Iwatani et al., 2007). Moreover, the analysis of endogenous reverse transcription in cell-free HIV-1 particles also indicated that $\mathrm{A} 3 \mathrm{G}$ reduces HIV-1 viral DNA levels by inhibiting the elongation of reverse transcription rather than by inducing the degradation of the reverse transcripts (Bishop et al., 2008). The block of RT elongation by A3G might be attributed to A3G's unique nucleic acid-binding ability (Iwatani et al., 2007). More recently, Wang et al. (2012) have observed physiological and functional interactions between RT and A3G, although our group 


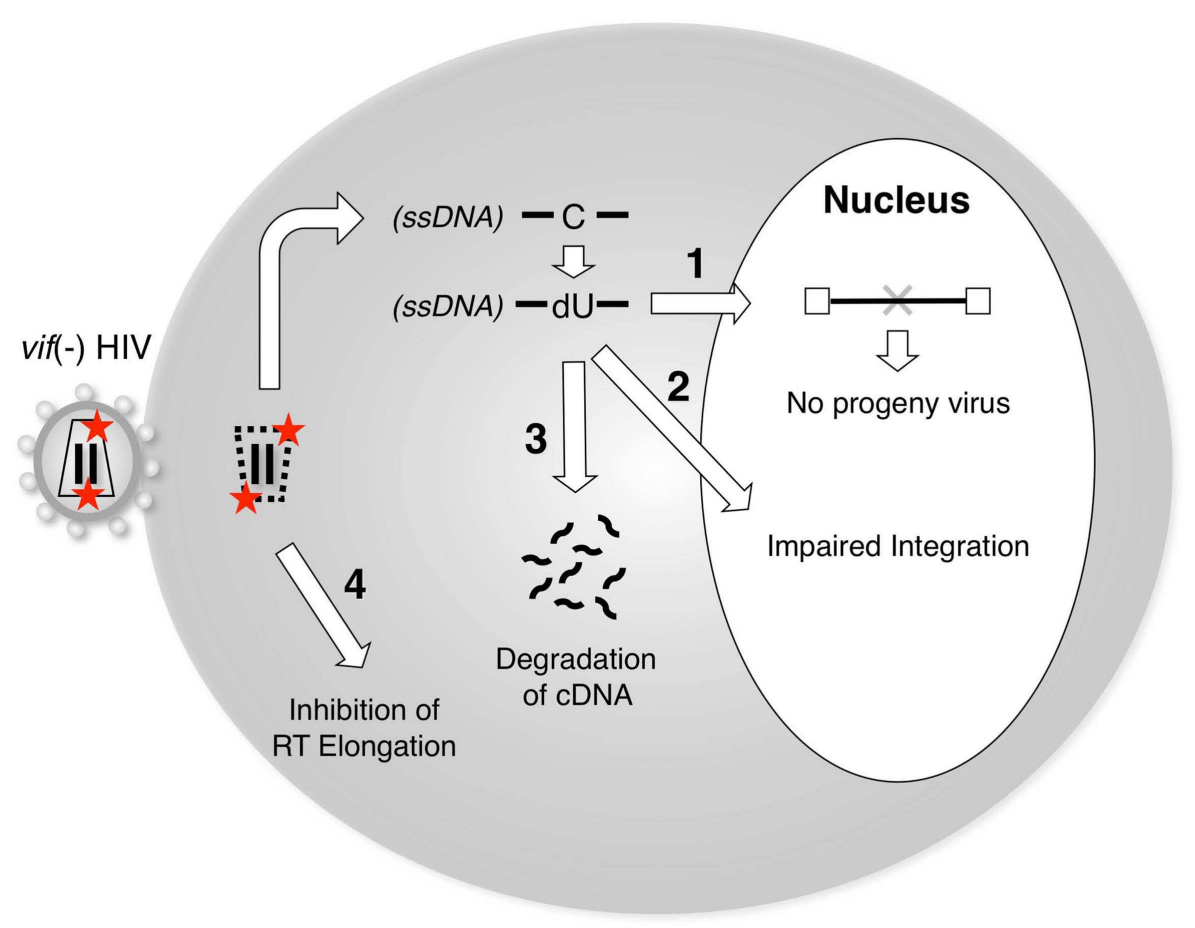

FIGURE 2 | A3G blocks the reverse transcription and/or integration of vif-deficient HIV-1. Packaging of A3G proteins into vif-deficient virus particles is prerequisite for the inhibition of viral replication by A3G. Upon the infection of target cells, A3G blocks the post-entry step of viral replication by one or more of the following mechanisms: (1) Cytidine deamination of nascent reverse transcripts by $A 3 G$ enzymes could prevent progeny virus production due to inactivating mutations in viral genes and/or proteins. (2)
A3G-mediated editing might create aberrant structures at viral DNA ends, which might be inefficient substrates for integration. (3) The reverse transcripts containing dU might induce DNA degradation by cellular DNA repair pathways. (4) RT-mediated elongation could be blocked by the presence of A3G on RNA or DNA templates. A3G might exert both deaminase activity-dependent (1-3) and deaminase activity-independent (4) functions to inhibit vif-deficient HIV-1 replication. has been unable to detect direct interactions using recombinant A3G and RT proteins (Iwatani, Y., and Levin, J. G., unpublished observations). It might be interesting to know whether direct interaction is applicable to other $\mathrm{A} 3$ proteins and/or retroviral RTs, i.e., how the broad range of A3G's inhibitory effect can be linked to the specific interaction between RT and A3G. Further investigations are required to understand the molecular mechanisms of the deaminase-independent pathway in more detail.

\section{CONCLUSIONS}

Studies over the past 10 years have established that human APOBEC3 family proteins potently restrict retroviral replication. However, the molecular mechanisms of the A3 family's antiviral activities remain unclear. Recent biochemical studies of A3G may provide a better understanding of these mechanisms. Currently, it

\section{REFERENCES}

Anant, S., and Davidson, N. O. (2000). An AU-rich sequence element (UUUN[A/U]U) downstream of the edited $\mathrm{C}$ in apolipoprotein $\mathrm{B}$ mRNA is a high-affinity binding site for Apobec-1: binding of Apobec1 to this motif in the $3^{\prime}$ untranslated region of c-myc increases mRNA stability. Mol. Cell. Biol. 20, 1982-1992.
Anant, S., MacGinnitie, A. J., and Davidson, N. O. (1995). apobec-1, the catalytic subunit of the mammalian apolipoprotein B mRNA editing enzyme, is a novel RNAbinding protein. J. Biol. Chem. 270, 14762-14767.

Anderson, J. L., and Hope, T. J. (2008). APOBEC3G restricts early HIV-1 replication in the cytoplasm of target cells. Virology 375, 1-12.

is possible that the deaminase activity of $\mathrm{A} 3 \mathrm{G}$ is largely required for its antiviral activity against vif-deficient HIV-1, although it is not known whether A3G-mediated deamination and/or the architecture of the catalytic center in $\mathrm{A} 3 \mathrm{G}$ are intrinsically required for its inhibitory activity. Further investigations will provide the fundamental answers to explain the first observation by von Schwedler et al., made when A3G had not yet been discovered.

\section{ACKNOWLEDGMENTS}

We thank Dr Judith G. Levin (NIH, NICHD) for helpful discussions. This work was supported in part by a grant-in-aid for scientific research from the Ministry of Education, Culture, Sports, Science, and Technology of Japan and by a grant for HIV/AIDS research from the Ministry of Health, Labor, and Welfare of Japan.

Beale, R. C., Petersen-Mahrt, S. K., Watt, I. N., Harris, R. S., Rada, C., and Neuberger, M. S. (2004). Comparison of the differential context-dependence of DNA deamination by APOBEC enzymes: correlation with mutation spectra in vivo. J. Mol. Biol. 337, 585-596.

Bennett, R. P., Salter, J. D., Liu, X., Wedekind, J. E., and Smith, H. C. (2008). APOBEC3G subunits self-associate via the C-terminal deaminase domain. J. Biol. Chem. 283, 33329-33336.

Bishop, K. N., Verma, M., Kim, E. Y., Wolinsky, S. M., and Malim, M. H. (2008). APOBEC3G inhibits elongation of HIV-1 reverse transcripts. PLoS Pathog. 4, e1000231. doi:10.1371/journal.ppat.1000231

Bogerd, H. P., Doehle, B. P., Wiegand, H. L., and Cullen, B. R. (2004). 
A single amino acid difference in the host APOBEC3G protein controls the primate species specificity of HIV type 1 virion infectivity factor. Proc. Natl. Acad. Sci. U.S.A. 101, 3770-3774.

Bogerd, H. P., Wiegand, H. L., Hulme, A. E., Garcia-Perez, J. L., O'Shea, K. S., Moran, J. V., and Cullen, B. R. (2006). Cellular inhibitors of long interspersed element 1 and Alu retrotransposition. Proc. Natl. Acad. Sci. U.S.A. 103, 8780-8785.

Bonvin, M., and Greeve, J. (2007). Effects of point mutations in the cytidine deaminase domains of $\mathrm{APOBEC} 3 \mathrm{~B}$ on replication and hypermutation of hepatitis B virus in vitro. J. Gen. Virol. 88, 3270-3274.

Bonvin, M., and Greeve, J. (2008). Hepatitis B: modern concepts in pathogenesis-APOBEC3 cytidine deaminases as effectors in innate immunity against the hepatitis B virus. Curr. Opin. Infect. Dis. 21, 298-303.

Browne, E. P., Allers, C., and Landau, N. R. (2009). Restriction of HIV-1 by APOBEC3G is cytidine deaminasedependent. Virology 387, 313-321.

Bulliard, Y., Turelli, P., Rohrig, U. F., Zoete, V., Mangeat, B., Michielin, O., and Trono, D. (2009). Functional analysis and structural modeling of human APOBEC3G reveal the role of evolutionarily conserved elements in the inhibition of human immunodeficiency virus type 1 infection and Alu transposition. J. Virol. 83, 12611-12621.

Burnett, A., and Spearman, P. (2007). APOBEC3G multimers are recruited to the plasma membrane for packaging into human immunodeficiency virus type 1 virus-like particles in an RNA-dependent process requiring the NC basic linker. J. Virol. 81, 5000-5013.

Chelico, L., Pham, P., Calabrese, P., and Goodman, M. F. (2006). APOBEC3G DNA deaminase acts processively $3^{\prime}->5^{\prime}$ on singlestranded DNA. Nat. Struct. Mol. Biol. 13, 392-399.

Chelico, L., Prochnow, C., Erie, D. A., Chen, X. S., and Goodman, M. F. (2010). Structural model for deoxycytidine deamination mechanisms of the HIV-1 inactivation enzyme APOBEC3G. J. Biol. Chem. 285, 16195-16205.

Chelico, L., Sacho, E. J., Erie, D. A., and Goodman, M. F. (2008). A model for oligomeric regulation of APOBEC3G cytosine deaminasedependent restriction of HIV. J. Biol. Chem. 283, 13780-13791.
Chen, K. M., Harjes, E., Gross, P. J., Fahmy, A., Lu, Y., Shindo, K., Harris, R. S., and Matsuo, H. (2008). Structure of the DNA deaminase domain of the HIV-1 restriction factor APOBEC3G. Nature 452, 116-119.

Cullen, B. R. (2003). HIV-1 Vif: counteracting innate antiretroviral defenses. Mol. Ther. 8, 525-527.

Feng, Y., and Chelico, L. (2011). Intensity of deoxycytidine deamination of HIV-1 proviral DNA by the retroviral restriction factor APOBEC $3 \mathrm{G}$ is mediated by the noncatalytic domain. J. Biol. Chem. 286, 11415-11426.

Fisher, A. G., Ensoli, B., Ivanoff, L., Chamberlain, M., Petteway, S., Ratner, L., Gallo, R. C., and WongStaal, F. (1987). The sor gene of HIV-1 is required for efficient virus transmission in vitro. Science 237, 888-893.

Fouchier, R. A., Simon, J. H., Jaffe, A. B., and Malim, M. H. (1996). Human immunodeficiency virus type 1 Vif does not influence expression or virion incorporation of gag-, pol-, and env-encoded proteins. J. Virol. 70, 8263-8269.

Friew, Y. N., Boyko, V., Hu, W. S., and Pathak, V. K. (2009). Intracellular interactions between APOBEC3G, RNA, and HIV-1 Gag: APOBEC3G multimerization is dependent on its association with RNA. Retrovirology $6,56$.

Goff, S. P. (2003). Death by deamination: a novel host restriction system for HIV-1. Cell 114, 281-283.

Guo, F., Cen, S., Niu, M., Saadatmand, J., and Kleiman, L. (2006). Inhibition of formula-primed reverse transcription by human APOBEC3G during human immunodeficiency virus type 1 replication. J. Virol. 80, 11710-11722.

Guo, F., Cen, S., Niu, M., Yang, Y., Gorelick, R. J., and Kleiman, L. (2007). The interaction of APOBEC3G with human immunodeficiency virus type 1 nucleocapsid inhibits tRNA3Lys annealing to viral RNA. J. Virol. 81, 11322-11331.

Haché, G., Liddament, M. T., and Harris, R. S. (2005). The retroviral hypermutation specificity of APOBEC $3 \mathrm{~F}$ and APOBEC3G is governed by the C-terminal DNA cytosine deaminase domain. J. Biol. Chem. 280, 10920-10924.

Harris, R. S., Bishop, K. N., Sheehy, A. M., Craig, H. M., Petersen-Mahrt, S. K., Watt, I. N., Neuberger, M. S., and Malim, M. H. (2003a). DNA deamination mediates innate immunity to retroviral infection. Cell 113, 803-809.
Harris, R. S., Sheehy, A. M., Craig, H. M. Malim, M. H., and Neuberger, M. S. (2003b). DNA deamination: not just a trigger for antibody diversification but also a mechanism for defense against retroviruses. Nat. Immunol. 4, 641-643.

Holden, L. G., Prochnow, C., Chang, Y. P., Bransteitter, R., Chelico, L., Sen, U., Stevens, R. C., Goodman, M. F., and Chen, X. S. (2008). Crystal structure of the anti-viral APOBEC3G catalytic domain and functional implications. Nature 456 121-124.

Holmes, R. K., Koning, F. A., Bishop, K. N., and Malim, M. H. (2007). APOBEC3F can inhibit the accumulation of HIV-1 reverse transcription products in the absence of hypermutation. Comparisons with APOBEC3G. J. Biol. Chem. 282, 2587-2595.

Huthoff, H., Autore, F., GalloisMontbrun, S., Fraternali, F., and Malim, M. H. (2009). RNA-dependent oligomerization of APOBEC3G is required for restriction of HIV1. PLoS Pathog. 5, e1000330. doi:10.1371/journal.ppat.1000330

Huthoff, H., and Malim, M. H. (2007). Identification of amino acid residues in APOBEC3G required for regulation by human immunodeficiency virus type 1 Vif and Virion encapsidation. J. Virol. 81, 3807-3815.

Iwatani, Y., Chan, D. S., Wang, F., Maynard, K. S., Sugiura, W., Gronenborn, A. M., Rouzina, I., Williams, M. C., Musier-Forsyth, K., and Levin, J. G. (2007). Deaminase-independent inhibition of HIV-1 reverse transcription by APOBEC3G. Nucleic Acids Res. 35 7096-7108.

Iwatani, Y., Takeuchi, H., Strebel, K. and Levin, J. G. (2006). Biochemical activities of highly purified, catalytically active human APOBEC3G: correlation with antiviral effect. J. Virol. 80, 5992-6002.

Jarmuz, A., Chester, A., Bayliss, J., Gisbourne, J., Dunham, I., Scott, J., and Navaratnam, N. (2002). An anthropoid-specific locus of orphan C to U RNA-editing enzymes on chromosome 22. Genomics 79, 285-296.

Kaiser, S. M., and Emerman, M. (2006). Uracil DNA glycosylase is dispensable for human immunodeficiency virus type 1 replication and does not contribute to the antiviral effects of the cytidine deaminase Apobec3G. J. Virol. 80, 875-882.

KewalRamani, V. N., and Coffin, J. M. (2003). Virology. Weapons of mutational destruction. Science 301, 923-925.

Kitamura, S., Ode, H., and Iwatani, Y. (2011). Structural features of antiviral APOBEC3 proteins are linked to their functional activities. Front. Microbiol. 2:258. doi:10.3389/fmicb.2011.00258

Langlois, M. A., and Neuberger, M. S. (2008). Human APOBEC3G can restrict retroviral infection in avian cells and acts independently of both UNG and SMUG1. J. Virol. 82, 4660-4664.

LaRue, R. S., Andresdottir, V., Blanchard, Y., Conticello, S. G., Derse, D. Emerman, M., Greene, W. C., Jonsson, S. R., Landau, N. R., Lochelt, M., Malik, H. S., Malim, M. H., Munk, C., O’Brien, S. J., Pathak, V. K., Strebel, K., Wain-Hobson, S., Yu, X. F., Yuhki, N., and Harris, R. S. (2009). Guidelines for naming nonprimate APOBEC3 genes and proteins. J. Virol. 83, 494-497.

Lavens, D., Peelman, F., Van der Heyden, J., Uyttendaele, I., Catteeuw, D., Verhee, A., Van Schoubroeck, B., Kurth, J., Hallenberger, S., Clayton, R., and Tavernier, J. (2010). Definition of the interacting interfaces of Apobec3G and HIV-1 Vif using MAPPIT mutagenesis analysis. Nucleic Acids Res. 38, 1902-1912.

Lecossier, D., Bouchonnet, F., Clavel, F., and Hance, A. J. (2003). Hypermutation of HIV-1 DNA in the absence of the Vif protein. Science 300, 1112

Levin, J. G., Guo, J., Rouzina, I., and Musier-Forsyth, K. (2005). Nucleic acid chaperone activity of HIV-1 nucleocapsid protein: Critical role in reverse transcription and molecular mechanism. Prog. Nucleic Acid Res. Mol. Biol. 80, 217-286.

Li, X. Y., Guo, F., Zhang, L., Kleiman, L., and Cen, S. (2007). APOBEC3G inhibits DNA strand transfer during HIV-1 reverse transcription. J. Biol. Chem. 282, 32065-32074.

Luo, K., Wang, T., Liu, B., Tian, C., Xiao, Z., Kappes, J., and Yu, X. F. (2007). Cytidine deaminases APOBEC $3 G$ and APOBEC3F interact with human immunodeficiency virus type 1 integrase and inhibit proviral DNA formation. J. Virol. 81, 7238-7248.

Mangeat, B., Turelli, P., Caron, G., Friedli, M., Perrin, L., and Trono, D. (2003). Broad antiretroviral defence by human APOBEC3G through lethal editing of nascent reverse transcripts. Nature 424, 99-103.

Mangeat, B., Turelli, P., Liao, S. and Trono, D. (2004). A single amino acid determinant governs the species-specific sensitivity of 
APOBEC3G to Vif action. J. Biol. Chem. 279, 14481-14483.

Mariani, R., Chen, D., Schrofelbauer, B., Navarro, F., Konig, R., Bollman, B., Munk, C., Nymark-McMahon, H., and Landau, N. R. (2003). Speciesspecific exclusion of APOBEC3G from HIV-1 virions by Vif. Cell 114, 21-31.

Marin, M., Rose, K. M., Kozak, S. L., and Kabat, D. (2003). HIV-1 Vif protein binds the editing enzyme APOBEC3G and induces its degradation. Nat. Med. 9, 1398-1403.

Mbisa, J. L., Barr, R., Thomas, J. A., Vandegraaff, N., Dorweiler, I. J., Svarovskaia, E. S., Brown, W. L., Mansky, L. M., Gorelick, R. J., Harris, R. S., Engelman, A., and Pathak, V. K. (2007). Human immunodeficiency virus type 1 cDNAs produced in the presence of APOBEC3G exhibit defects in plus-strand DNA transfer and integration. J. Virol. 81, 7099-7110.

McDougall, W. M., Okany, C., and Smith, H. C. (2011). Deaminase activity on single-stranded DNA (ssDNA) occurs in vitro when APOBEC3G cytidine deaminase forms homotetramers and higher-order complexes. J. Biol. Chem. 286, 30655-30661.

Miyagi, E., Brown, C. R., Opi, S., Khan, M., Goila-Gaur, R., Kao, S., Walker, R. C. Jr., Hirsch, V., and Strebel, K. (2010). Stably expressed APOBEC3F has negligible antiviral activity. $J$. Virol. 84, 11067-11075.

Miyagi, E., Schwartzkopff, F., Plishka, R., Buckler-White, A., Clouse, K. A., and Strebel, K. (2008). APOBEC3Gindependent reduction in virion infectivity during long-term HIV1 replication in terminally differentiated macrophages. Virology 379, 266-274.

Muckenfuss, H., Hamdorf, M., Held, U., Perkovic, M., Lower, J., Cichutek, K., Flory, E., Schumann, G. G., and Munk, C. (2006). APOBEC3 proteins inhibit human LINE-1 retrotransposition. J. Biol. Chem. 281, 22161-22172.

Narvaiza, I., Linfesty, D. C., Greener, B. N., Hakata, Y., Pintel, D. J., Logue, E., Landau, N. R., and Weitzman, M. D. (2009). Deaminase-independent inhibition of parvoviruses by the APOBEC3A cytidine deaminase. PLoS Pathog. 5, e1000439. doi:10.1371/journal.ppat.1000439

Navaratnam, N., Bhattacharya, S., Fujino, T., Patel, D., Jarmuz, A. L., and Scott, J. (1995). Evolutionary origins of apoB mRNA editing: catalysis by a cytidine deaminase that has acquired a novel RNA-binding motif at its active site. Cell 81, 187-195.

Navarro, F., Bollman, B., Chen, H., König, R., Yu, Q., Chiles, K., and Landau, N. R. (2005). Complementary function of the two catalytic domains of APOBEC3G. Virology 333, 374-386.

Nguyen, D. H., Gummuluru, S., and $\mathrm{Hu}$, J. (2007). Deaminationindependent inhibition of hepatitis $B$ virus reverse transcription by APOBEC3G. J. Virol. 81, 4465-4472.

Okeoma, C. M., Lovsin, N., Peterlin, B. M., and Ross, S. R. (2007). APOBEC3 inhibits mouse mammary tumour virus replication in vivo. Nature 445, 927-930.

Pace, C., Keller, J., Nolan, D., James, I., Gaudieri, S., Moore, C., and Mallal, S. (2006). Population level analysis of human immunodeficiency virus type 1 hypermutation and its relationship with APOBEC3G and vif genetic variation. J. Virol. 80, 9259-9269.

Russell, R. A., Smith, J., Barr, R., Bhattacharyya, D., and Pathak, V. K. (2009). Distinct domains within APOBEC3G and APOBEC3F interact with separate regions of human immunodeficiency virus type 1 Vif. J. Virol. 83, 1992-2003.

Salter, J. D., Krucinska, J., Raina, J., Smith, H. C., and Wedekind, J. E. (2009). A hydrodynamic analysis of APOBEC3G reveals a monomer-dimer-tetramer selfassociation that has implications for anti-HIV function. Biochemistry 48, 10685-10687.

Schrofelbauer, B., Chen, D., and Landau, N. R. (2004). A single amino acid of APOBEC3G controls its speciesspecific interaction with virion infectivity factor (Vif). Proc. Natl. Acad. Sci. U.S.A. 101, 3927-3932.

Shandilya, S. M., Nalam, M. N., Nalivaika, E. A., Gross, P. J., Valesano, J. C., Shindo, K., Li, M., Munson, M., Royer, W. E., Harjes, E., Kono, T., Matsuo, H., Harris, R. S., Somasundaran, M., and Schiffer, C. A. (2010). Crystal structure of the APOBEC3G catalytic domain reveals potential oligomerization interfaces. Structure $18,28-38$.

Sheehy, A. M., Gaddis, N. C., Choi, J. D., and Malim, M. H. (2002). Isolation of a human gene that inhibits HIV-1 infection and is suppressed by the viral Vif protein. Nature 418, 646-650.

Sheehy, A. M., Gaddis, N. C., and Malim, M. H. (2003). The antiretroviral enzyme APOBEC3G is degraded by the proteasome in response to HIV-1 Vif. Nat. Med. 9, 1404-1407.
Shlyakhtenko, L. S., Lushnikov, A. Y., Li, M., Lackey, L., Harris, R. S., and Lyubchenko, Y. L. (2011). Atomic force microscopy studies provide direct evidence for dimerization of the HIV restriction factor APOBEC3G. J. Biol. Chem. 286, 3387-3395.

Shubsda, M. F., Paoletti, A. C., Hudson, B. S., and Borer, P. N. (2002). Affinities of packaging domain loops in HIV-1 RNA for the nucleocapsid protein. Biochemistry 41, 5276-5282.

Simon, V., Zennou, V., Murray, D., Huang, Y., Ho, D. D., and Bieniasz, P. D. (2005). Natural variation in Vif: differential impact on APOBEC3G/3F and a potential role in HIV-1 diversification. PLoS Pathog. 1, e6. doi:10.1371/journal.ppat.0010006

Sova, P., and Volsky, D. J. (1993). Efficiency of viral DNA synthesis during infection of permissive and nonpermissive cells with vifnegative human immunodeficiency virus type 1. J. Virol. 67, 6322-6326.

Stenglein, M. D., and Harris, R. S. (2006). APOBEC3B and APOBEC3F inhibit L1 retrotransposition by a DNA deamination-independent mechanism. J. Biol. Chem. 281, 16837-16841.

Stopak, K., de Noronha, C., Yonemoto, W., and Greene, W. C. (2003). HIV1 Vif blocks the antiviral activity of APOBEC3G by impairing both its translation and intracellular stability. Mol. Cell 12, 591-601.

Strebel, K., Daugherty, D., Clouse, K., Cohen, D., Folks, T., and Martin, M. A. (1987). The HIV 'A' (sor) gene product is essential for virus infectivity. Nature 328, 728-730.

Suspène, R., Sommer, P., Henry, M., Ferris, S., Guétard, D., Pochet, S., Chester, A., Navaratnam, N., WainHobson, S., and Vartanian, J. P. (2004). APOBEC3G is a singlestranded DNA cytidine deaminase and functions independently of HIV reverse transcriptase. Nucleic Acids Res. 32, 2421-2429.

Svarovskaia, E. S., Xu, H., Mbisa, J. L., Barr, R., Gorelick, R. J., Ono, A., Freed, E. O., Hu, W. -S., and Pathak, V. K. (2004). Human apolipoprotein B mRNA-editing enzyme-catalytic polypeptide-like 3G (APOBEC3G) is incorporated into HIV-1 virions through interactions with viral and nonviral RNAs. J. Biol. Chem. 279, 35822-35828.

Takeda, E., Tsuji-Kawahara, S., Sakamoto, M., Langlois, M. A., Neuberger, M. S., Rada, C., and Miyazawa, M. (2008). Mouse
APOBEC3 restricts friend leukemia virus infection and pathogenesis in vivo. J. Virol. 82, 10998-11008.

Turelli, P., Mangeat, B., Jost, S., Vianin, S., and Trono, D. (2004). Inhibition of hepatitis B virus replication by APOBEC3G. Science 303, 1829.

von Schwedler, U., Song, J., Aiken, C., and Trono, D. (1993). vif is crucial for human immunodeficiency virus type 1 proviral DNA synthesis in infected cells. J. Virol. 67, 4945-4955.

Wang, X., Ao, Z., Chen, L., Kobinger, G., Peng, J., and Yao, X. (2012). The cellular antiviral protein APOBEC3G interacts with HIV-1 reverse transcriptase and inhibits its function during viral replication. J. Virol. 86, 3777-3786.

Wedekind, J. E., Dance, G. S., Sowden, M. P., and Smith, H. C. (2003) Messenger RNA editing in mammals: new members of the APOBEC family seeking roles in the family business. Trends Genet. 19, 207-216.

Wedekind, J. E., Gillilan, R., Janda, A., Krucinska, J., Salter, J. D., Bennett, R. P., Raina, J., and Smith, H. C. (2006). Nanostructures of APOBEC3G support a hierarchical assembly model of high molecular mass ribonucleoprotein particles from dimeric subunits. J. Biol. Chem. 281, 38122-38126.

Wissing, S., Montano, M., Garcia-Perez, J. L., Moran, J. V., and Greene, W. C. (2011). Endogenous APOBEC3B restricts LINE-1 retrotransposition in transformed cells and human embryonic stem cells. J. Biol. Chem. 286, 36427-36437.

Xu, H., Svarovskaia, E. S., Barr, R., Zhang, Y., Khan, M. A., Strebel, K., and Pathak, V. K. (2004). A single amino acid substitution in human APOBEC3G antiretroviral enzyme confers resistance to HIV-1 virion infectivity factor-induced depletion. Proc. Natl. Acad. Sci. U.S.A. 101, 5652-5657.

Yang, B., Chen, K., Zhang, C., Huang, S., and Zhang, H. (2007). Virionassociated uracil DNA glycosylase-2 and apurinic/apyrimidinic endonuclease are involved in the degradation of APOBEC3G-edited nascent HIV-1 DNA. J. Biol. Chem. 282, 11667-11675.

Yu, Q., König, R., Pillai, S., Chiles, K., Kearney, M., Palmer, S., Richman, D., Coffin, J. M., and Landau, N. R. (2004). Single-strand specificity of APOBEC3G accounts for minus-strand deamination of the HIV genome. Nat. Struct. Mol. Biol. 11, 435-442. 
Yu, X., Yu, Y., Liu, B., Luo, K., Kong, W., Mao, P., and Yu, X. F. (2003). Induction of APOBEC3G ubiquitination and degradation by an HIV-1 Vif-Cul5-SCF complex. Science 302, 1056-1060.

Zhang, H., Yang, B., Pomerantz, R. J., Zhang, C., Arunachalam, S. C., and Gao, L. (2003). The cytidine deaminase CEM15 induces hypermutation in newly synthesized HIV-1 DNA. Nature 424, 94-98.
Zhang, L., Saadatmand, J., Li, X., Guo, F., Niu, M., Jiang, J., Kleiman, L., and Cen, S. (2008). Function analysis of sequences in human APOBEC3G involved in Vifmediated degradation. Virology 370, 113-121.

Conflict of Interest Statement: The authors declare that the research was conducted in the absence of any commercial or financial relationships that could be construed as a potential conflict of interest.

Received: 29 May 2012; accepted: 21 June 2012; published online: 09 July 2012. Citation: Imahashi $M$, Nakashima $M$ and Iwatani Y (2012) Antiviral mechanism and biochemical basis of the human APOBEC3 family. Front. Microbio. 3:250. doi: 10.3389/fmicb.2012.00250
This article was submitted to Frontiers in Virology, a specialty of Frontiers in Microbiology.

Copyright (๑) 2012 Imahashi, Nakashima and Iwatani. This is an open-access article distributed under the terms of the Creative Commons Attribution License, which permits use, distribution and reproduction in other forums, provided the original authors and source are credited and subject to any copyright notices concerning any third-party graphics etc. 